\title{
Bird Identification by Image Recognition
}

\author{
${ }^{1}$ Madhuri A. Tayal, ${ }^{* 2}$ Atharva Mangrulkar, ${ }^{3}$ Purvashree Waldey, ${ }^{4}$ Chitra Dangra \\ ${ }^{1-4}$ Asst. Prof., Department of Information Technology, Shri Ramdeobaba College of Engineering and Management, Nagpur \\ Email:tayalma@rknec.edu,mangrulkarap@rknec.edu,waldeyps@rknec.edu,dangrach@rknec.edu
}

Received: $20^{\text {th }}$ September 2018, Accepted: $11^{\text {th }}$ October 2018, Published: $31^{\text {st }}$ October 2018

\begin{abstract}
Bird watching is an art of observing, studying and researching on birds. People who pursue bird watching as a hobby or passion can be termed as bird-watchers and others involved in scientific study and research on birds are termed as ornithologists. All ornithologists are bird-watchers but not all bird-watchers are ornithologists.

Accurate bird-identification is an important aspect of bird-watching. In India, not many software are available that use input image for identification of a specie, thus making the identification process cumbersome for bird-watchers. To overcome this issue, we came across a solution of building software as a project. The database consisted of 300-400 different images of number of bird species. Concept of transfer learning and pre-trained algorithm made feature extraction possible from an image. The result obtained were of high efficiency as the software could easily identify a bird species from an image whose dataset was present in the database.
\end{abstract}

\section{Keywords}

Image Recognition, Bird Identification, Transfer Learning, Feature Extraction, Support Vector Machine, Machine Learning, MATLAB

\section{Introduction}

Many people across countries are getting into this interest of bird-watching as a hobby or extra-curricular activity. In modern world, it acts as a great stress buster and a cheap way of getting connected with nature. Another benefit of birdwatching is awareness about nature conservation by observing behavior, migratory pattern, population, and conservation status of bird species. From conservation point of view, it is important that more and more number of people turn towards bird-watching and help collect data that can be used to study birds.

Sometimes, bird identification can be difficult for beginners as well as experienced bird-watchers. The focus of our project is to also simplify the identification process. As far as India is concerned, there is no bird identification software available that takes image as an input and gives the identity of the bird as output. There are bird identification software available for countries such as US and Canada (eg. eBird, Merlin Bird ID) but very few good quality bird identification software are available in India.

For beginners, the software will be of great help because at the early stages of bird-watching, identifying or differentiating between different species can be difficult and frustrating. To experienced bird-watchers also this software will majorly help in identification.

\section{Materials and Methods}

Following are the commonly used bird identification software in India:

Indian Birds:

This application covers all the common bird species found in India. It offers a quiz about birds to increase the user's knowledge about bird identification and displays the names and information in Indian languages.[1]

Eguide to Birds of the Indian Subcontinent:

This application not only covers birds found in India but also Pakistan, Nepal, Bhutan, Sri Lanka, Maldives, and Bangladesh. It covers a lot of local birds but the information has not been updated since 2013 and costs Rs. 1800. [2]

Database collection:

We downloaded first 200 results of google images in bulk using Google Chrome extension for each species. We segregated images in different folders and named the folder after every species names. Folder name is taken as label while training and testing.

\section{A. Pretrained Model: AlexNet}

AlexNet is a pre-trained algorithm for image recognition. It has been trained to classify thousands of images of daily-life objects like pencil, keyboard, animals etc. The classified images are segregated under different labels given by the user. The network has learned feature extraction for a large number of images. It takes image as an input and gives the label to 
Helix Vol. 8(6): 4349- 4352

which the object in the image matches, as an output. It also gives the probabilities for each object category. [3]

AlexNet algorithm required 100-200 images of same bird species of same pixel size, as a database. Specifically, it required $227 * 227$ pixel images for training and testing. The compression of images can be done manually or by using a image compression software. We used Caesium software for compressing images in bulk.

\section{B. Applying Transfer Learning: Feature Extraction}

In the experimental setup of our project, the features specific to our dataset by using AlexNet model. The model was not trained to identify birds as an object in the image. We applied transfer learning to fine-tune the model to identify birds. The pre-trained network is used as a starting point to learn a new task. The learned features are transferred using few training images. [3]

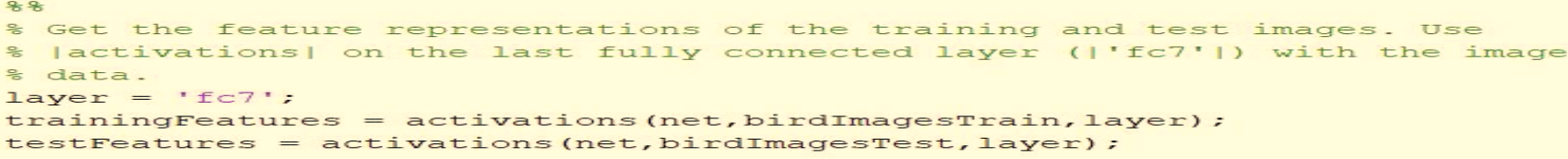

Figure 1: Feature Representation

\section{Classification of Images: SVM Classifier}

SVM (Support Vector Machine) which is a supervised machine learning algorithm is used for classification. Features extracted will be categorized according to the labels with the help of SVM.

a. $\quad$ Fit Image Classifier: The features extracted from the training images are used as predictor variables and are used to fit a multiclass. For this purpose, we implemented SVM (Support Vector Machine) using fitcecoc function which is a Statistics and Machine Learning toolbox. [4]

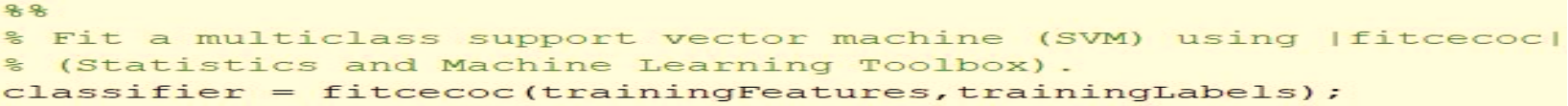

Figure 2: fitcecoc Function

b. Classify Test Images: Now, we need to classify the test images. This is done by the trained SVM model on the basis of feature extracted from the test images. [4]

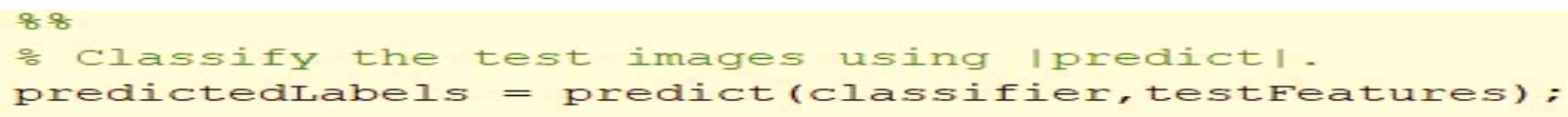

Figure 3: predict Function

\section{Accuracy:}

Accuracy is the fraction of labels predicted by the model correctly. If the accuracy is not high enough using feature extraction, transfer learning can be used.

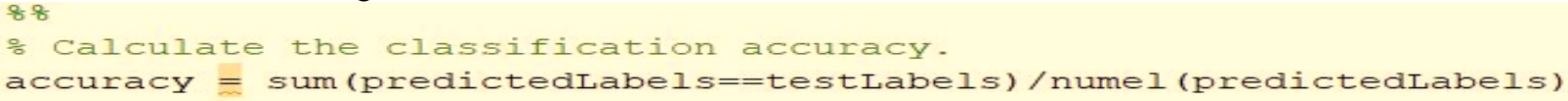

\section{Figure 4: Calculating Accuracy for given Input Image}

\section{Results and Discussion}

When we run the code, the dataset is divided into training and testing. First, it trains the system and then tests for accuracy then asks for user input. It gives the label as a result with input image.

Following are the steps of execution: 


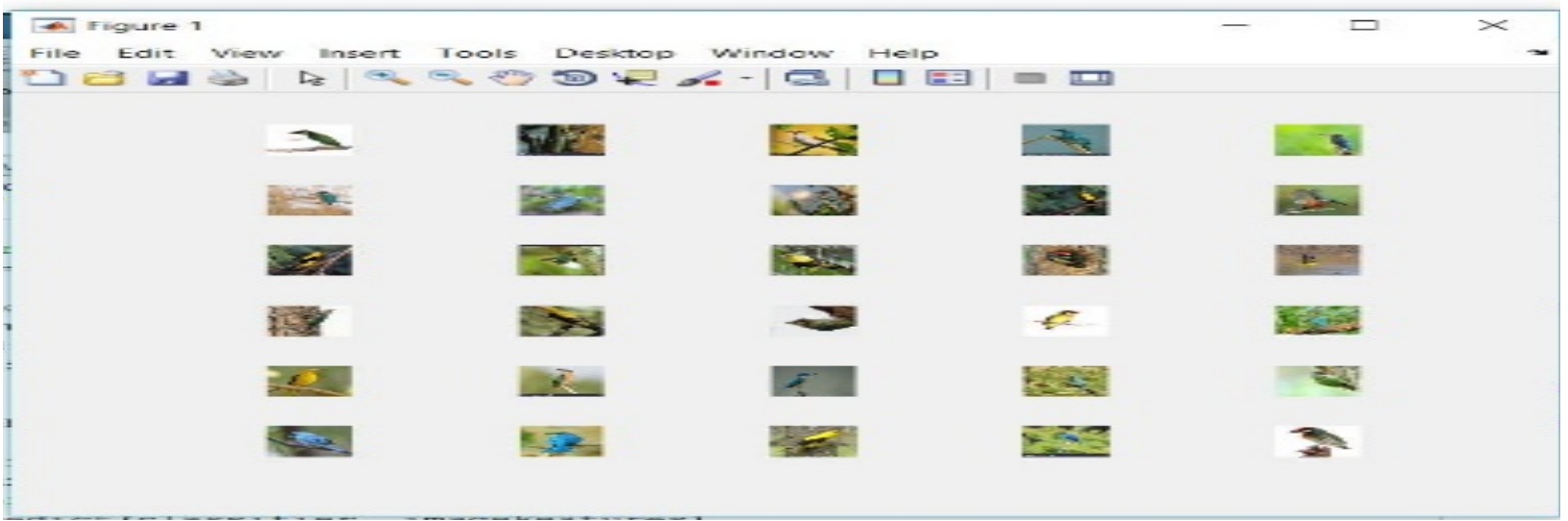

Figure 5: Display of Trained Images

Few trained images of each bird species present in the dataset are displayed in the above figure.

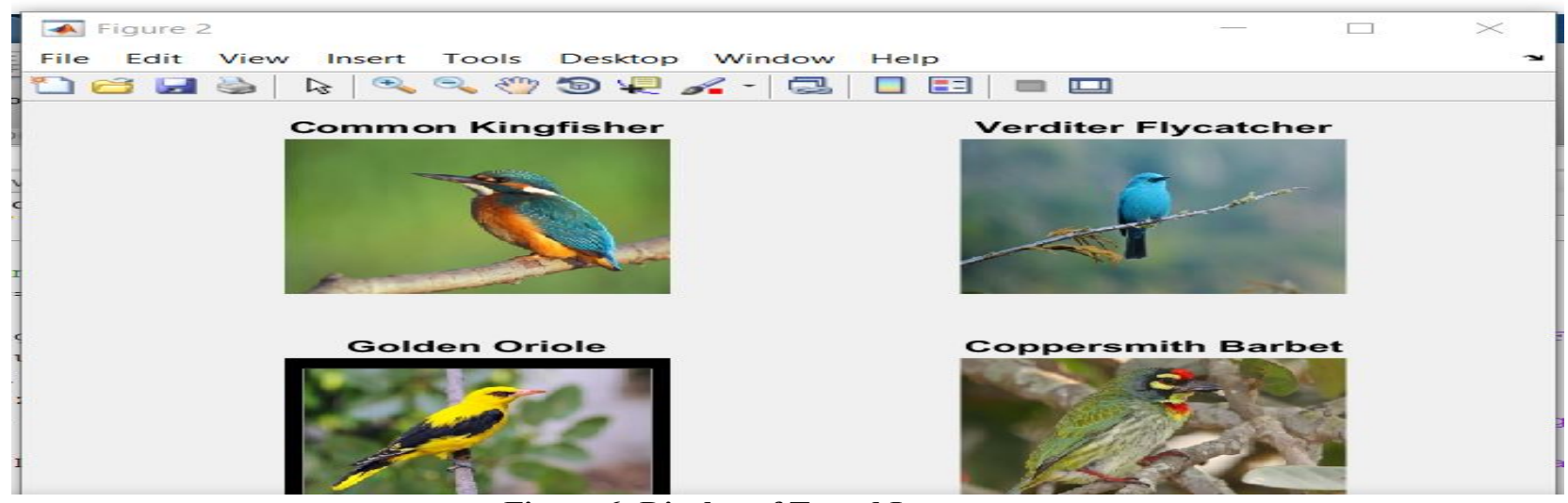

Figure 6: Display of Tested Images

Then the tested images are displayed. Figure 6 displays image of each species along with its label (species name).

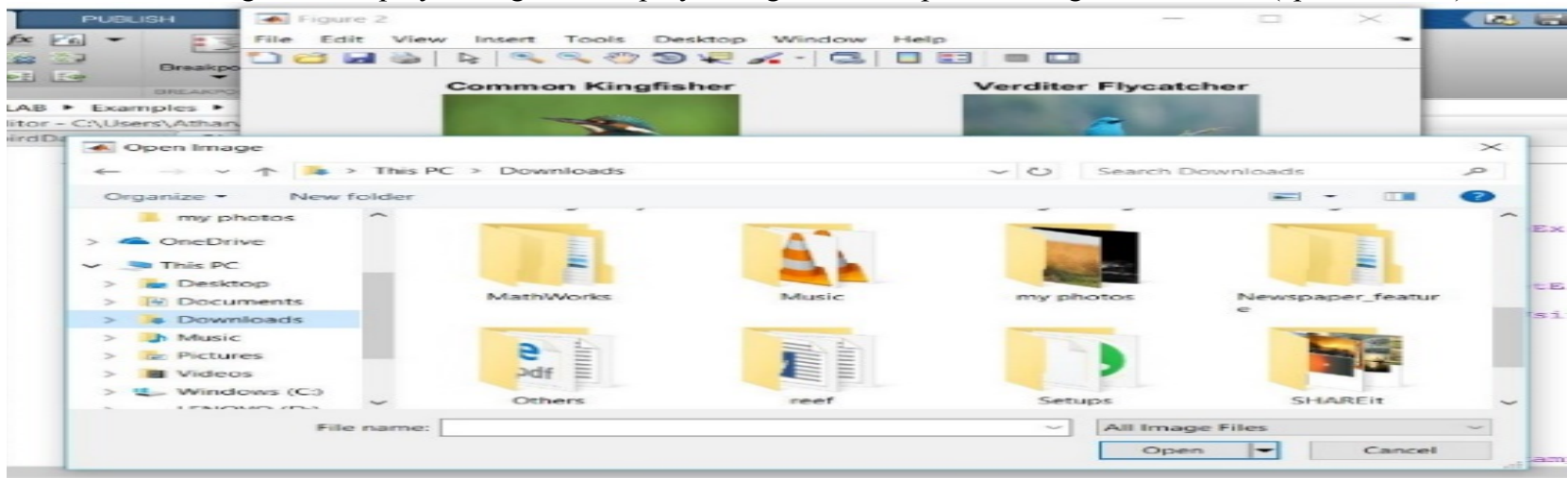

Figure 7: Inserting User Input 


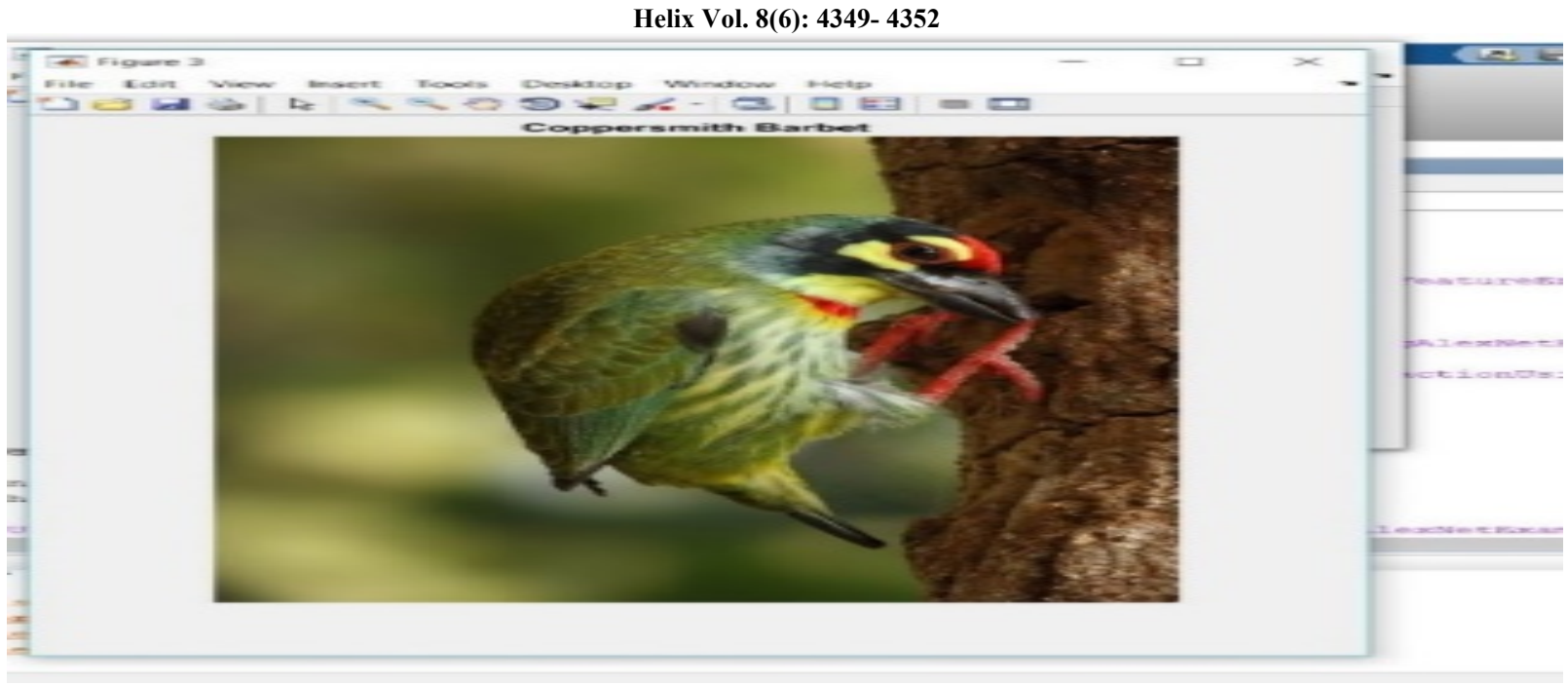

Figure 8: Output: Species Name and Input Image

Bird image in the above figure was given as input. It is photographed by authors and was not included in training and testing database.

\begin{tabular}{|l|l|l|l|}
\hline Sr. No. & $\begin{array}{l}\text { Label name } \\
\text { (Species) }\end{array}$ & $\begin{array}{l}\text { Accurately identified images / } \\
\text { Tested images }\end{array}$ & $\begin{array}{l}\text { Accurately identified images } \\
\text { (\%) }\end{array}$ \\
\hline 1 & Coppersmith Barbet & $10 / 10$ & $100 \%$ \\
\hline 2 & Golden Oriole & $8 / 10$ & $80 \%$ \\
\hline 3 & Verditer Flycatcher & $7 / 10$ & $70 \%$ \\
\hline 4 & Common Kingfisher & $9 / 10$ & $90 \%$ \\
\hline & Total & $\mathbf{3 4 / 4 0}$ & $\mathbf{8 5 \%}$ \\
\hline
\end{tabular}

Table 1: Efficiency Table

\section{Conclusion}

The main idea behind developing the identification software is to build awareness regarding bird-watching, bird and their identification, especially birds found in India. It also caters to need of simplifying bird identification process and thus making bird-watching easier. The technology used in the experimental setup is Transfer learning in MATLAB on a pretrained algorithm (AlexNet). It uses feature extraction for image recognition. The method used is good enough to extract features and classify images.

This paper presents a summary of our project. The main purpose of the project is to identify the bird species from an image given as input by the user. The technology used is transfer learning and MATLAB. We used MATLAB because it is suitable for implementing advanced algorithm and gives good numerical precision accuracy. It is also general purpose and scientific. We achieved an accuracy of $80 \%-85 \%$. We believe this project extends a great deal of scope as the purpose meets. In wildlife research and monitoring, this concept can be implemented in camera traps to maintain the record of wildlife movement in specific habitat and behavior of any species.

\section{Acknowledgement}

We sincerely thank Dr. Madhuri Tayal, Asst. Prof., Dept. of Information Technology for her guidance and encouragement in carrying out this project. Any errors are our own and should not tarnish the reputations of this esteemed person. We also wish to thank Ms. Pratyaksha Jha, 4th year, Dept. of Electronics \& Communication for her guidance and help at every step of our research work.

\section{References}

[1] Indian Birds [Online] https://play.google.com/store/apps/details?id=com.kokanes.birdsinfo\&hl=en [2] Bird Watching Apps: Five Useful Apps to Get Started With Birding [Online] https://gadgets.ndtv.com/apps/features/bird-watching-apps-five-useful-apps-to-get-started-with-birding-1640679

[3] Transfer learning using Alex Net [Online] https://in.mathworks.com/help/nnet/examples/transfer-learning-usingalexnet.html

[4] Feature extraction using AlexNet [Online] https://in.mathworks.com/help/nnet/examples/feature-extraction-usingalexnet.html\#d119e4167 